\title{
REGULATION OF AGGRECANASES FROM THE ADAMTS FAMILY AND AGGRECAN NEOEPITOPE FORMATION DURING IN VITRO CHONDROGENESIS OF HUMAN MESENCHYMAL STEM CELLS
}

\author{
S. Boeuf ${ }^{1}$, F. Graf ${ }^{1}$, J. Fischer ${ }^{1}$, B. Moradi ${ }^{1}$, C. B. Little² and W. Richter ${ }^{1 *}$ \\ ${ }^{1}$ Research Centre for Experimental Orthopaedics, Orthopaedic University Hospital, Heidelberg, Germany \\ ${ }^{2}$ Raymond Purves Bone and Joint Research Laboratories, Kolling Institute of Medical Research, \\ University of Sydney at The Royal North Shore Hospital, Sydney, Australia
}

\begin{abstract}
Aggrecanases from the ADAMTS (A Disintegrin And Metalloproteinase with ThromboSpondin motifs) family are important therapeutic targets due to their essential role in aggrecan depletion in arthritic diseases. Whether their function is also important for matrix rearrangements during chondrogenesis and thus, cartilage regeneration, is however so far unknown. The aim of this study was to analyse the expression and function of ADAMTS with aggrecanase activity during chondrogenic differentiation of human mesenchymal stem cells (MSCs). Chondrogenic differentiation was induced in bone marrow-derived MSC pellets and expression of COL2A1, aggrecan, ADAMTS1, $4,5,9,16$ and furin was followed by quantitative RTPCR. Formation of the NITEGE (ADAMTS-cleaved) and DIPEN (MMP-cleaved) aggrecan neoepitopes was detected by immunohistochemistry. While the expression of ADAMTS4, 9, 16 and furin was up-regulated during chondrogenesis, ADAMTS1 and 5 were down-regulated. Despite this regulation of ADAMTS, no formation of NITEGE neoepitopes occurred in MSC pellets, indicating no ADAMTS-induced cleavage of aggrecan. In contrast, MMP-induced cleavage of aggrecan appeared at $14 \mathrm{~d}$ after induction of chondrogenesis. Submission of differentiated MSC pellets to IL1 $\beta$ treatment for $3 \mathrm{~d}$ resulted in strong upregulation of ADAMTS1, 4 and 5, rapid proteoglycan depletion, and stimulation of ADAMTS-induced but not MMP-induced cleavage of aggrecan. Thus, there is no evidence for ADAMTS-induced aggrecan cleavage during chondrogenesis, but proteoglycan turnover is rapidly inducible under inflammatory signals. Therapeutic aggrecanase inhibition for treatment of arthritic disease may thus not impede regenerative self-healing pathways based on chondrogenesis of local progenitor cells in the joint.
\end{abstract}

Keywords: Mesenchymal stem cells; regenerative medicine; aggrecanase; proteoglycan depletion; aggrecan neoepitopes; cartilage development; inflammation; interleukin $1 \beta$

*Address for correspondence:

Wiltrud Richter, PhD

Research Centre for Experimental Orthopaedics

Orthopaedic University Hospital Heidelberg

Schlierbacher Landstrasse 200a

69118 Heidelberg, Germany

Telephone: +496221969253

Fax: +49 6221969288

E-mail: wiltrud.richter@med.uni-heidelberg.de

\section{Introduction}

Chondrogenesis is a process engaging differentiating cells into production of a large amount of extracellular matrix which fills the growing distance between them. This matrix is mainly composed of a network of collagen type II fibrils and of proteoglycans, among which aggrecan is predominant. Matrix homeostasis is ensured as chondrocytic cells synthesise matrix degrading enzymes such as matrix metalloproteases (MMPs) and enzymes from the "A Disintegrin And Metalloproteinase with ThromboSpondin motifs" protein family (ADAMTS). Matrix rearrangements by cleavage of collagen fibrils or aggrecan are expected to be particularly important during chondrogenesis, when the extracellular space is growing. The analysis of mouse models has indeed indicated that MMP function influences foetal cartilage development and specifically endochondral ossification (Little and Fosang, 2010). MMPs are strongly regulated during the chondrogenesis of human mesenchymal stem cells (MSCs) and we have shown previously that their inhibition prevents chondrogenic differentiation in vitro (Bertram et al., 2009). This suggests that MMP activity is essential for chondrogenic differentiation of adult human MSCs. In contrast, the function of aggrecanases from the ADAMTS family does not appear to influence foetal cartilage development in mice (Little and Fosang, 2010). Only a few data on the expression and activity of aggrecanases during chondrogenic differentiation of human MSCs are available so far. Expression profiles of the two main aggrecanases, ADAMTS4 and 5, in MSC pellets until $21 \mathrm{~d}$ of chondrogenesis suggested a regulation of both genes during this process, with the functional implications remaining unknown (Djouad et al., 2007).

Loss of aggrecan is an early feature of cartilage degeneration in osteoarthritis (OA) (Aigner and McKenna, 2002). Aggrecan can be cleaved by both MMPs and ADAMTS at different sites. However, it has been demonstrated that ADAMTS proteases and not MMPs are the major proteases responsible for aggrecan depletion during the progression of OA (Malfait et al., 2002; Sumer et al., 2007). Inhibiting ADAMTS cleavage of the aggrecan interglobular domain improves restoration of cartilage aggrecan after its depletion in inflammatory joint disease, while the inhibition of MMP cleavage does not (Little et al., 2007). More particularly, it has been suggested that ADAMTS4 and 5, also known as aggrecanases 1 and 2, are the two major contributors to aggrecan depletion in humans (Song et al., 2007) while in mice ADAMTS5 appears to be the single enzyme involved 
(Stanton et al., 2005). ADAMTS proteases, thus, play an essential role in matrix homeostasis of cartilage and, in the case of OA, in its destruction (Jones et al., 2005). They have for this reason gained attention as potential therapeutic targets in OA (Fosang and Little, 2008). In the context of therapeutic ADAMTS inhibition, it is of interest to examine the role these enzymes might play during in vitro chondrogenic differentiation of MSCs, since possible pathways of in vivo regeneration based on chondrogenesis of endogenous progenitor cells should not be impaired. While MMP inhibitors blocked in vitro chondrogenesis of human MSCs, it could be an advantage of aggrecanase inhibitors if their chondroprotective effect would not impair regeneration. The aim of this study therefore was to characterise the expression and activity of aggrecanases from the ADAMTS protein family during the chondrogenic differentiation of MSCs.

The ADAMTS form a family of secreted proteases which include 19 human genes (Porter et al., 2005). Phylogenetic analysis identifies closely related ADAMTS for which aggrecanase activity has been demonstrated. These include ADAMTS 1, 4, 5 and 8 (Porter et al., 2005). Aggrecanase activity has furthermore been demonstrated for the structurally less related ADAMTS9 and ADAMTS16 (Zeng et al., 2006). Of these aggrecanases, all but one, ADAMTS8, have been shown to be expressed in cartilage (Kevorkian et al., 2004). Therefore, the genes of ADAMTS1, 4, 5, 9 and 16 have been included in this study. Common to all ADAMTS is a signal peptide at the $\mathrm{N}$ terminus followed by a prodomain including at least one cleavage site for the proprotein peptidase furin. ADAMTS are expressed as zymogens and activated through furin cleavage either intracellularly in the endoplasmic reticulum (Longpre and Leduc, 2004; Wang et al., 2004), at the cell surface (Koo et al., 2006) or extracellularly (Longpre et al., 2009). For this reason, furin expression in MSCs was analysed in parallel.

\section{Materials and Methods}

\section{Tissue samples}

Human OA articular cartilage samples were obtained from 7 patients (age from 47 to 88 ) undergoing total knee replacement surgery. Healthy cartilage samples were obtained from the knees of 6 patients (age from 30 to 52) undergoing amputation for diseases not affecting the knee joint. Cartilage was carefully removed from the tibial plateau and femoral condyles, washed with phosphate buffered saline (PBS) to avoid contamination by other cells and snap-frozen in liquid nitrogen. Bone marrow samples for isolation of MSCs were obtained from 6 patients (age from 10 to 65) undergoing total hip replacement or iliac bone graft harvest. The studies were approved by the local ethics committee and informed consent was obtained from all individuals included in the study.

\section{Isolation and expansion of MSCs}

MSCs were isolated from fresh bone marrow samples as described previously (Haynesworth et al., 1992; Winter et al., 2003). Briefly, cells were fractionated on a FicollPaque Plus density gradient (GE Healthcare, Munich, Germany), and the low-density MSC-enriched fraction was washed and seeded in culture flasks in an expansion medium (Reyes et al., 2001; Winter et al., 2003) consisting of DMEM high glucose, $40 \%$ MCDB201, $2 \%$ foetal calf serum (FCS), $2 \times 10^{-8} \mathrm{M}$ dexamethasone, $10^{-4} \mathrm{M}$ ascorbic acid-2-phosphate, $10 \mu \mathrm{g} / \mathrm{mL}$ insulin, $10 \mu \mathrm{g} / \mathrm{mL}$ transferrin, $10 \mathrm{ng} / \mathrm{mL}$ sodium selenite, $100 \mathrm{units} / \mathrm{mL}$ penicillin, $100 \mu \mathrm{g} /$ $\mathrm{mL}$ streptomycin, $10 \mathrm{ng} / \mathrm{mL}$ recombinant epidermal growth factor (Miltenyi Biotec, Bergisch Gladbach, Germany) and $10 \mathrm{ng} / \mathrm{mL}$ platelet-derived growth factor BB (Active Bioscience, Hamburg, Germany). Non-adherent material was removed after 24-48 h. During expansion, medium was replaced twice a week. Cells were replated at $5 \times 10^{3}$ cells $/ \mathrm{cm}^{2}$ and used at passage 3 .

\section{Chondrogenic differentiation of MSCs and IL1及 treatment}

For induction of chondrogenesis at high cell density in three-dimensional culture, pellets consisting of $5 \times 10^{5}$ expanded MSCs were formed by centrifugation at $300 \mathrm{~g}$ (Eppendorf, Hamburg, Germany). Pellets were exposed to chondrogenic medium (DMEM high glucose with $0.1 \mu \mathrm{M}$ dexamethasone, $0.17 \mathrm{mM}$ ascorbic acid-2-phosphate, $5 \mu \mathrm{g} /$ $\mathrm{mL}$ insulin, $5 \mu \mathrm{g} / \mathrm{mL}$ transferrin, $5 \mathrm{ng} / \mathrm{mL}$ selenous acid, $1 \mathrm{mM}$ sodium pyruvate, $0.35 \mathrm{mM}$ proline, $1.25 \mathrm{mg} / \mathrm{mL}$ BSA, 100 units $/ \mathrm{mL}$ penicillin and $100 \mu \mathrm{g} / \mathrm{mL}$ streptomycin) supplemented with $10 \mathrm{ng} / \mathrm{mL}$ recombinant human TGF- $\beta 1$ (PeproTech, Hamburg, Germany) and cultivated for 6 weeks. Medium was changed every 2 to $3 \mathrm{~d}$. After $42 \mathrm{~d}$ of chondrogenic induction, some pellets were maintained 3 additional days in chondrogenic medium without TGF $\beta$ $\pm 10 \mathrm{ng} / \mathrm{mL}$ IL1 $\beta$ (Promokine, Heidelberg, Germany). Medium was not changed during these $3 \mathrm{~d}$.

Table 1. List of oligonucleotides used for quantitative RT-PCR analysis

\begin{tabular}{lll}
\hline Gene & Forward primer (5' to 3') & Reverse primer (5' to 3') \\
\hline ACTB & CTCTTCCAGCCTTCCTTCC & CGATCCACACGGAGTACTTG \\
COL2A1 & TGGCCTGAGACAGCATGAC & AGTGTTGGGAGCCAGATTGT \\
ACAN & GCACCATGCCTTCTGCTT & GGAACCACTTGGGTCACG \\
ADAMTS1 & GAACAAAACCGACAGAAAGCA & TGTCACATTCCCTCATCGTG \\
ADAMTS4 & AGGCACTGGGCTACTAC & GGGATAGTGACCACATTGTT \\
ADAMTS5 & TCTAAGCCCTGGTCCAAATG & TCGTGGTAGGTCCAGCAAA \\
ADAMTS9 & ATCCATCCATAATGGCTTCC & CCTGGAGTATCGTGCAGTCA \\
ADAMTS16 & GAGGATAGCCGTAATGTTTG & CTTGAATTAGCGATGACCTG \\
FURIN & TGTGGTGTAGGTGTGGCCTA & TGGATGTGGTTGGGGTTC \\
\hline
\end{tabular}




\section{RNA isolation}

3-4 pellets per donor and group were pooled, minced and total RNA was isolated using a standard guanidinium thiocyanate/phenol extraction protocol (peqGOLD Trifast, PeqLab, Erlange, Germany). Polyadenylated mRNA was then purified from total RNA with oligo-d(T)-coupled magnetic beads (Dynabeads, Invitrogen, Karlsruhe, Germany) according to the manufacturer's instructions. For the isolation of RNA from cartilage samples, shock-frozen tissue was pulverised mechanically and consecutively dissolved in lysis/binding buffer for direct poly $(\mathrm{A})^{+}-\mathrm{mRNA}$ isolation using oligo-d(T)-coupled beads (Dynabeads, Invitrogen).

\section{Quantitative RT-PCR}

mRNA was subjected to first strand cDNA synthesis using reverse transcriptase (Sensiscript, Qiagen, Hilden, Germany) and oligo-d(T) primers. Expression levels of individual genes were analysed by quantitative PCR (Lightcycler, Roche). Aliquots of first-stranded cDNAs were amplified using gene-specific primer sets (Table 1) obtained from Eurofins (Ebersberg, Germany) and real-time fluorimetric intensity of SYBR green I was monitored. The Cycling parameters were optimised according to the Light-Cycler protocol from Roche (Light-Cycler Operator's Manual, Version 3.5, October 2000). The apparent threshold cycles (Ct) of the genes of interest were compared to the $\mathrm{Ct}$ of the housekeeping gene $\beta$-actin (ACTB) in the same cDNA sample. A standard curve based on diluted samples of ACTB cDNA was prepared by qPCR to deduce the e correlation factor between cDNA concentration and differences of $\mathrm{Ct}(\Delta \mathrm{Ct})$ of ACTB. Changes of ACTB concentration could be estimated as $\mathrm{e}^{\wedge}(0.5756 \times \Delta \mathrm{Ct})$. This exponential function was then used to calculate fold differences between the gene of interest ( $\mathrm{Ct}$ of the gene of interest, $\mathrm{Ct}-\mathrm{GO}$ ) and ACTB (Ct of ACTB, Ct-ACTB) in the same sample, which were converted as $\%$ of ACTB expression. The calculated expression level in $\%$ of $\mathrm{ACTB}$ was thus equal to:

$$
100 \times \mathrm{e}^{\wedge}(0.5756 \times(\mathrm{Ct}-\mathrm{ACTB}-\mathrm{Ct}-\mathrm{GO}))
$$

All gene expression levels shown in figures and tables refer to relative levels in \% of ACTB calculated with this formula. Melting curves and agarose gel electrophoresis of the PCR products were used for quality control.

\section{Histology}

MSC Pellets were fixed in $4 \%$ paraformaldehyde, as described (Hennig et al., 2007). All samples were embedded in paraffin. For visualisation of proteoglycans, $5 \mu \mathrm{m}$ sections were stained with $2 \mathrm{~g} / \mathrm{L}$ safranin $\mathrm{O}$ (Schmid. Köngen, Germany) and counterstained with fast green (Sigma-Aldrich, Seelze, Germany). Immunohistological staining for collagen type II was performed as described (Winter et al., 2003). Briefly, sections were incubated with a monoclonal mouse anti-human collagen II antibody (clone II-4C11; 1:1000; MP Biomedicals, Eschwege, Germany). Reactivity was detected using biotinylated goat anti-mouse antibody (Dianova, Hamburg, Germany), streptavidin- alkaline phosphatase (Dako, Glostrup, Denmark), and fast red. Immunostaining for aggrecan neoepitopes generated by ADAMTS or MMP cleavage of the interglobular domain was done as previously described (Smith et al., 2008). Briefly, $5 \mu \mathrm{m}$ paraffin sections were rehydrated, digested with 0.1 units $/ \mathrm{mL}$ protease-free chondroitinase ABC, (Sigma-Aldrich, Castle Hill, Australia) and 0.1 units/ $\mathrm{mL}$ of keratanase I (Sapphire Biosciences, Alexandria, Australia), prior to overnight incubation with primary antibodies to the aggrecanase-generated aggrecan neoepitope NITEGE $(0.55 \mu \mathrm{g} / \mathrm{mL}$; mAb Agg-C1; provided by Dr. Carl Flannery, Pfizer Inc, Cambridge, MA, USA), and the MMP-generated aggrecan neoepitope DIPEN ( 0.17 $\mu \mathrm{g} / \mathrm{mL}$; affinity-purified polyclonal antisera; provided by Assoc. Prof Amanda Fosang, University of Melbourne, Melbourne, Australia). Equivalent concentrations of species-matched immunoglobulins on identically treated sections were used as a negative control.

\section{Quantification of proteoglycan content}

Differentiated MSC pellets ( 2 or 3 per donor) were washed with PBS and mechanically crushed in $0.5 \mathrm{~mL}$ guanidine hydrochloride $(\mathrm{GuHCl})$ extraction buffer $(4 \mathrm{M} \mathrm{GuHCl} / 100$ $\mathrm{mM}$ Tris, $\mathrm{pH} 8.5)$. After incubation $\left(30 \mathrm{~min}, 4{ }^{\circ} \mathrm{C}\right)$ and centrifugation (13000 rpm, $15 \mathrm{~min}, 4{ }^{\circ} \mathrm{C}$ ), extracted proteoglycan in the supernatants was quantified with alcian blue following Björnsson (1993). In brief, chondroitin sulphate $(3.125-200 \mu \mathrm{g} / \mathrm{mL})$ was used as a standard. $50 \mu \mathrm{L}$ SAT buffer $\left(0.3 \% \mathrm{H}_{2} \mathrm{SO}_{4} ; 0.75 \%\right.$ Triton X-100 in distilled $\mathrm{H}_{2} \mathrm{O}$ ) was added to $100 \mu \mathrm{L}$ supernatant before shaking for $15 \mathrm{~min}$. Then, $750 \mu \mathrm{L}$ alcian blue working solution (1 volume alcian blue stock solution (3\% (w/v) alcian blue (Carl Roth, Karlsruhe, Germany) in $0.1 \% \mathrm{H}_{2} \mathrm{SO}_{4}, 0.4 \mathrm{M}$ $\mathrm{GuHCl})+5$ volumes SAT buffer +9 volumes $\mathrm{H}_{2} \mathrm{O}$ ) was added. After $30 \mathrm{~min}$ at room temperature under shaking, the precipitate was harvested by centrifugation ( $15 \mathrm{~min}, 13,000$ rpm, RT) and the supernatant was discarded. The pellet was washed in $500 \mu \mathrm{L}$ DMSO buffer (40\% DMSO; $0.05 \mathrm{M}$ $\mathrm{MgCl}_{2}$ in distilled $\mathrm{H}_{2} \mathrm{O}$ ), kept at room temperature for 15 $\mathrm{min}$, centrifuged (15 $\mathrm{min}, 20,000 \mathrm{~g}$ ) and the supernatant was again discarded. In the following step the pellet was dissolved in $500 \mu \mathrm{L}$ guanidine/propanol buffer (4 M GuHCl, $33 \%$ n-propanol; $0.25 \%$ Triton X-100). Two aliquots $(200 \mu \mathrm{L})$ were transferred to 96 well flat bottom plates and absorbance was measured at $650 \mathrm{~nm}$.

\section{Statistics}

Median values and standard deviation were calculated for all variables. For comparison of time points during chondrogenesis, Friedmann Test with post-hoc Wilcoxon Signed-Rank Tests was conducted. Only significant Friedmann Tests were analysed post-hoc and results without Bonferroni correction are mentioned in the results section. The Wilcoxon test was furthermore applied for the analysis of MSC treated with or without IL1 $\beta$ while the Mann-Whitney test was used for differences between cartilage samples and MSCs. A two-tailed significance value $p<0.05$ was considered significant. Data analysis was performed with SPSS for Windows 16.0 (SPSS Inc., Chicago, IL, USA). 
A
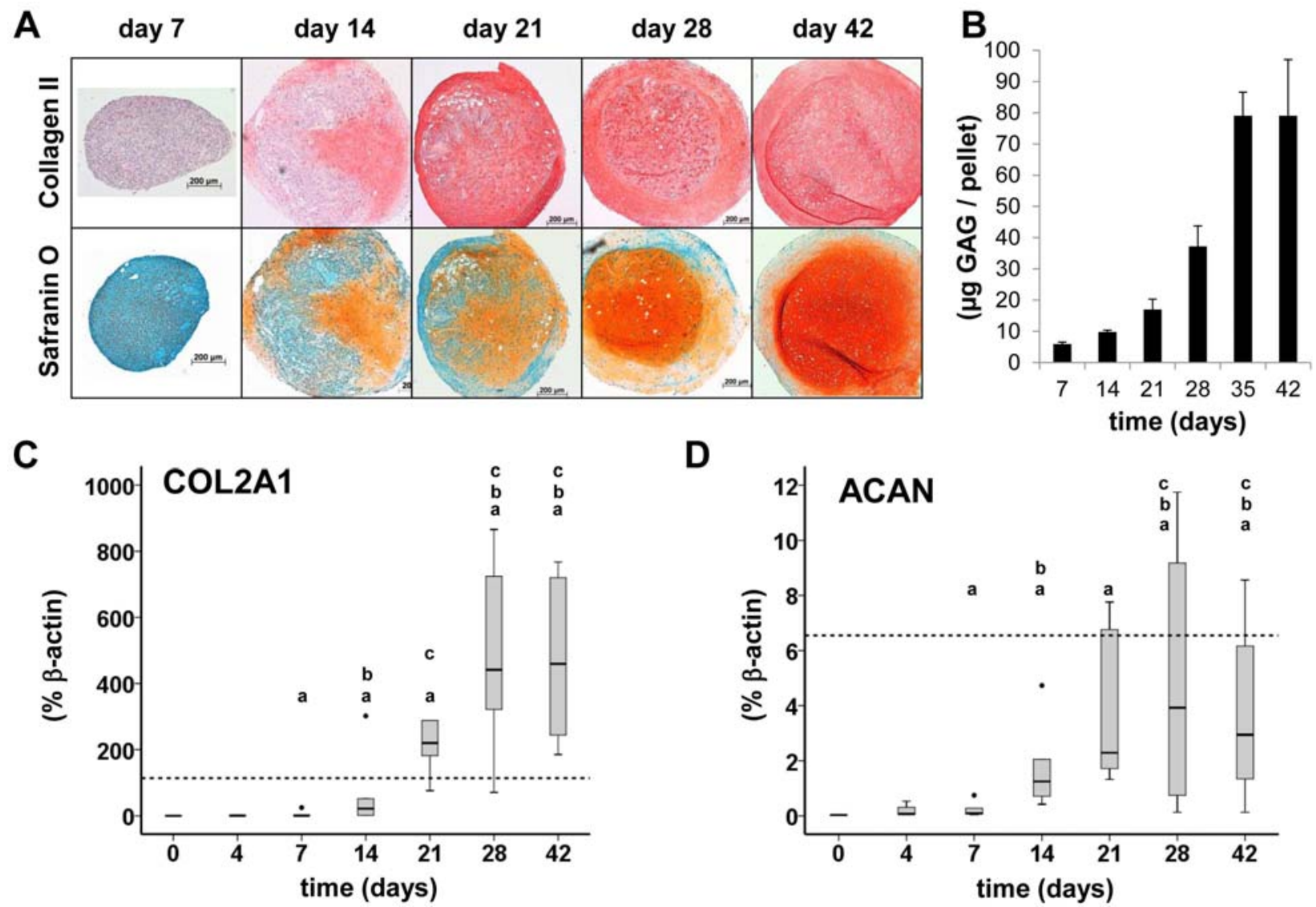

Fig. 1. Matrix production during the chondrogenic differentiation of MSCs. (A) Paraffin sections of MSC pellets were analysed 7, 14, 21, 28 and $42 \mathrm{~d}$ after induction of chondrogenesis. Production of type II collagen was determined by immunohistochemical analysis and accumulation of proteoglycans by Safranin O staining. Pellets from one representative donor are shown. (B) Proteoglycan content was quantified separately from 3 pellets from 1 representative donor harvested at day 7, 14, 21, 35 and 42 after chondrogenic induction. Gene expression levels of COL2A1 (C) and aggrecan (D) were measured by quantitative RT-PCR in undifferentiated MSCs (day 0) and after 4, 7, 14, 21, 28 and $42 \mathrm{~d}$ of chondrogenic induction (MSC from $n=6$ donors per time point). Gene expression levels were quantified with $\beta$-actin as the reference housekeeping gene. For each time point, the median expression ratio in \% in MSC samples is represented by a solid black line, the boxes represent the interquartile range (IQR) extending between the 25 th and 75 th percentile and the whiskers extend to a maximum of 1.5 IQR. Black dots are outlier values. Broken horizontal lines represent the median relative gene expression of the respective genes in normal cartilage samples $(n=6)$. Statistical analysis is based on the non-parametric Wilcoxon test $(p<0.05)$. $\mathbf{a}=$ significantly different from day $0 ; \mathbf{b}=$ significantly different from day $7 ; \mathbf{c}=$ significantly different from day 14 .

\section{Results}

\section{Upregulation of aggrecan during chondrogenic differentiation of MSCs}

Chondrogenesis was induced in pellets of MSCs from 6 donors under supplementation with TGF $\beta 1$. As evidenced by immunohistological analysis for collagen type II and staining of proteoglycans with safranin $\mathrm{O}$, first accumulation of a cartilage-like matrix in part of the pellet was detected at day 14 (Fig. 1A). The pellets of all donors showed similar patterns of collagen type II and proteoglycan accumulation and exhibited full chondrogenic differentiation after $42 \mathrm{~d}$. The expression of COL2A1 and aggrecan (ACAN) mRNA increased to significantly higher levels than at day 0 as early as the first week of pellet culture in differentiation medium (Figs. 1C and D). Thereafter, the expression of both COL2A1 and
ACAN progressively increased from day 7 to 14 (24.8fold, and 13.7-fold, respectively; $p=0.043$ for both) and from day 14 to 28 (20.3-fold, and 3.13-fold, respectively; $p=0.043$ for both). There was no further change in either COL2A1 or ACAN expression between day 28 and day 42 . GAG content in MSC pellets was analysed for one donor and showed the expected up-regulation in accordance with safranin $\mathrm{O}$ staining which was lagging behind the up-regulation of ACAN gene expression (Fig. 1B). Levels were increasing until day 35 and there was no further change between day 35 and 42 .

\section{Regulation of ADAMTS expression during chondrogenesis of MSCs}

Gene expression levels of aggrecanases during chondrogenesis of MSCs were assessed by quantitative RT-PCR analysis of mRNA levels of ADAMTS1, 4, 5, 9 
Table 2. Median values of gene expression levels as \% of ACTB expression in normal cartilage, OA cartilage, MSCs before differentiation and MSCs $42 \mathrm{~d}$ after chondrogenic induction ( $n=6$ donors).

\begin{tabular}{lrllllllllllll}
\hline Gene & \multicolumn{3}{c}{ normal } & \multicolumn{3}{c}{ OA } & \multicolumn{4}{c}{ MSC, day 0 } & \multicolumn{3}{c}{ MSC, day 42 } \\
\hline COL2A1 & 114.34 & \pm & 211.90 & $\mathbf{9 2 2 . 4 1 *}$ & \pm & $\mathbf{3 5 3 . 4 2}$ & $\mathbf{0 . 0 6 *}$ & \pm & $\mathbf{0 . 0 5}$ & $\mathbf{4 5 9 . 7 0 *}$ & \pm & $\mathbf{2 3 8 . 0 6}$ \\
ACAN & 6.55 & \pm & 4.83 & 8.26 & \pm & 4.03 & $\mathbf{0 . 0 3 *}$ & \pm & $\mathbf{0 . 0 1}$ & 2.94 & \pm & 3.14 \\
ADAMTS1 & 3.95 & \pm & 2.46 & 2.71 & \pm & 2.27 & 3.35 & \pm & 1.38 & $\mathbf{0 . 3 3 *}$ & \pm & $\mathbf{0 . 2 5}$ \\
ADAMTS4 & 1.04 & \pm & 1.90 & 0.72 & \pm & 0.56 & 0.05 & \pm & 0.02 & 0.37 & \pm & 0.35 \\
ADAMTS5 & 2.48 & \pm & 5.25 & 2.04 & \pm & 1.02 & $\mathbf{0 . 3 0} *$ & \pm & $\mathbf{0 . 1 1}$ & $\mathbf{0 . 1 0 *}$ & \pm & $\mathbf{0 . 0 8}$ \\
ADAMTS9 & 0.94 & \pm & 1.00 & 0.59 & \pm & 0.45 & $\mathbf{0 . 1 2} *$ & \pm & $\mathbf{0 . 0 3}$ & 0.53 & \pm & 0.19 \\
ADAMTS16 & 0.02 & \pm & 0.03 & 0.10 & \pm & 0.13 & 0.01 & \pm & 0.01 & $\mathbf{0 . 0 9 *}$ & \pm & $\mathbf{0 . 0 2}$ \\
FURIN & 0.63 & \pm & 0.56 & $\mathbf{2 . 2 7 *}$ & \pm & $\mathbf{0 . 6 0}$ & $\mathbf{0 . 2 9} *$ & \pm & $\mathbf{0 . 1 2}$ & 1.04 & \pm & 0.48 \\
\hline
\end{tabular}

* Significantly different from normal, Wilcoxon test, $p<0.05$

and 16 , and of the proprotein peptidase furin at day $0,4,7$, $14,21,28$ and 42 after induction of chondrogenesis in MSC pellet cultures $(n=6)$. Furthermore, in order to compare expression levels detected in MSCs to physiological levels in cartilage, gene expression was determined in normal healthy cartilage samples from 6 donors and in OA cartilage samples from 7 donors (Table 2).

The median gene expression of ADAMTS 1 in MSCs declined 10 -fold over the period of $42 \mathrm{~d}$ of chondrogenic differentiation from $3.35 \%$ in undifferentiated MSCs at day 0 to $0.33 \%$ on day 42 (Fig. 2A). After stable gene expression levels comparable to expression in cartilage during the first week, ADAMTS1 expression dropped between day 7 and 14 and thereafter remained at levels significantly lower than at day $0(p=0.043)$. Among the other genes analysed, only ADAMTS5 showed a similar temporal change in expression profile (Fig. 2B), although ADAMTS5 was initially more than 8-fold lower expressed in undifferentiated MSCs than in normal cartilage ( $p=$ 0.002). A 3-fold drop of ADAMTS5 expression levels occurred between day 7 and 14 , corresponding to a statistically significant decline between day 0 and day $42(p=0.028)$. Remarkably, this decline occurred during the main shift of ACAN upregulation. In comparison to ADAMTS5, the other classical aggrecanase, ADAMTS4, showed a different regulation pattern (Fig. 2C). Expression was low in undifferentiated MSCs and showed a more than 40-fold increase on day 4. After this transient peak, gene expression levels stabilised to levels close to expression in cartilage and significantly higher than at day 0 (7fold at day $42 ; p=0.028$ ). Similarly gene expression of ADAMTS9 was upregulated during chondrogenesis (Fig. 2D), increasing significantly from day 0 to day 42 (4-fold; $p=0.028$ ), reaching levels not significantly different from normal cartilage and very close to OA cartilage (Table 2). ADAMTS16 was essentially not expressed in undifferentiated MSCs (levels lower than $0.01 \%$ for all donors on day 0 ) and showed a significant increase to $0.14 \%$ after $14 \mathrm{~d}$ of chondrogenic differentiation compared to day $0(p=0.028)$ (Fig. $2 \mathrm{E}$ ). ADAMTS16 was the only aggrecanase with higher median expression levels in OA cartilage compared to normal cartilage, a difference which almost reached significance (Table 2; $p=0.051)$, in agreement with previously published data (Kevorkian et al., 2004). ADAMTS16 expression levels at day 42 after chondrogenesis were similar to OA cartilage and significantly higher than in normal cartilage (5.5fold; $p=0.009$ ). The mRNA of the proprotein peptidase furin increased significantly from day 0 to day 7 of chondrogenesis $(p=0.043)$ (Fig. 2F) and reached a 4-fold increase after $42 \mathrm{~d}(p=0.043)$. Furin showed a significant 3.58-fold higher expression in OA cartilage compared to normal cartilage $(p=0.017)$, and at day 42 in pellet culture of MSCs, expression was similar to levels in normal cartilage and significantly lower than in OA cartilage (2.14-fold; $p=0.004)$. ADAMTS4, 9, 16 and furin were thus all up-regulated during chondrogenesis of MSCs and reached levels comparable to OA cartilage (ADAMTS4, 9 and 16) or normal cartilage (ADAMTS4, 9 and furin) after differentiation. This indicates that these aggrecanases could possibly be activated during the differentiation process of MSCs when ACAN deposition is initiated.

\section{Aggrecan proteolysis during the chondrogenesis of MSCs}

In order to determine whether aggrecanase activity can be detected during chondrogenic differentiation of MSCs, an immunohistochemical analysis of ACAN neo-epitopes in MSC pellets was performed. Neo-epitopes occurring after cleavage of ACAN by ADAMTS (NITEGE epitope) or by MMPs (DIPEN epitope) were analysed in MSC pellets at day 14, 21, 28, 35, and 42 after induction of chondrogenesis (Fig. 3). All ADAMTS analysed in this study have been shown to produce NITEGE neo-epitopes (Porter et al., 2005; Zeng et al., 2006). While the DIPEN neo-epitope was detected from day 14 and increased progressively during differentiation, no NITEGE neo-epitopes were apparent in MSC pellets at any time. This suggests that MMP cleavage but not ADAMTS cleavage of ACAN occurs at detectable levels during chondrogenic differentiation of MSCs.

\section{Rapid loss of proteoglycan induced by IL1B}

While aggrecanases such as ADAMTS4, 9 and 16 were expressed in chondrogenic MSCs at similar levels to in cartilage, no ADAMTS-induced cleavage of ACAN was detected. In order to examine whether aggrecanase activity of ADAMTS is inducible in MSC pellets, the ADAMTS expression and activity was analysed in chondrogenic MSC pellets under an inflammatory signal. MSC pellets after $42 \mathrm{~d}$ of chondrogenic induction were incubated for $3 \mathrm{~d}$ in the same medium deprived of TGF $\beta$ and supplemented with $10 \mathrm{ng} / \mathrm{mL}$ IL $1 \beta$. The IL1 $\beta$ treatment induced a rapid 

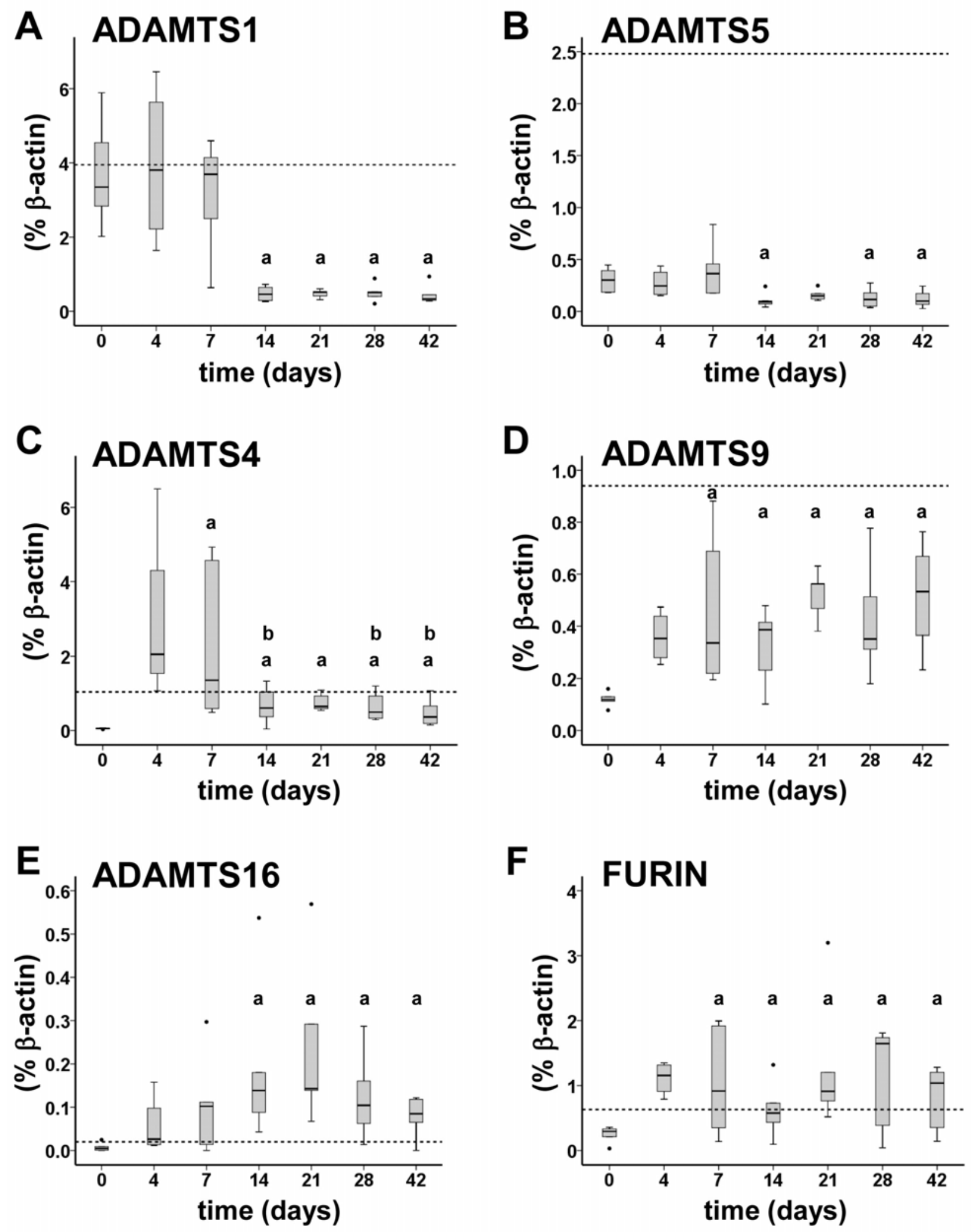

Fig. 2. Expression of ADAMTS1 (A), ADAMTS5 (B), ADAMTS4 (C), ADAMTS9 (D), ADAMTS16 (E) and FURIN (F) during the chondrogenic differentiation of MSCs. Gene expression levels as assessed by real time PCR are presented as relative percentage values compared to the housekeeping gene $\beta$-actin in the respective samples. Samples from 6 donors were analysed at each time point, except for day $4(n=4)$. For each time point, the median expression ratio in MSC samples is represented by a solid black line, the boxes represent the interquartile range (IQR) extending between the 25th and 75th percentile and the whiskers extend to a maximum of 1.5 IQR. Black dots are outlier values. Broken horizontal lines represent the median relative gene expression of the respective genes in normal cartilage samples $(n=6)$. Statistical analysis is based on the non-parametric Wilcoxon test $(p<0.05)$. a $=$ significantly different from day $0 ; \mathbf{b}=$ significantly different from day 7 . 


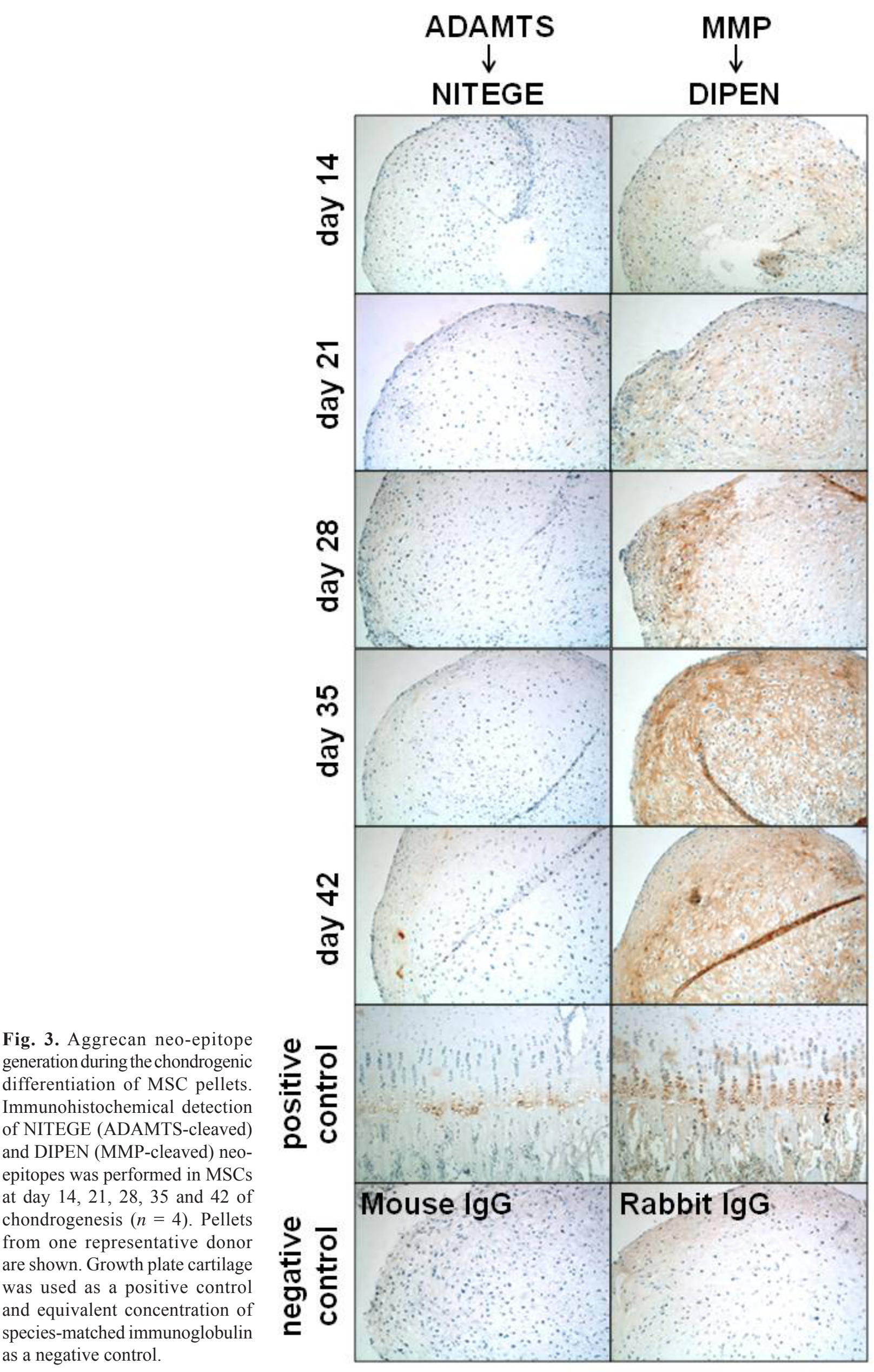

Fig. 3. Aggrecan neo-epitope generation during the chondrogenic differentiation of MSC pellets Immunohistochemical detection of NITEGE (ADAMTS-cleaved) and DIPEN (MMP-cleaved) neoat day 14, 21, 28, 35 and 42 of chondrogenesis $(n=4)$. Pellets from one representative donor are shown. was used as a positive control as a negative control. 
A

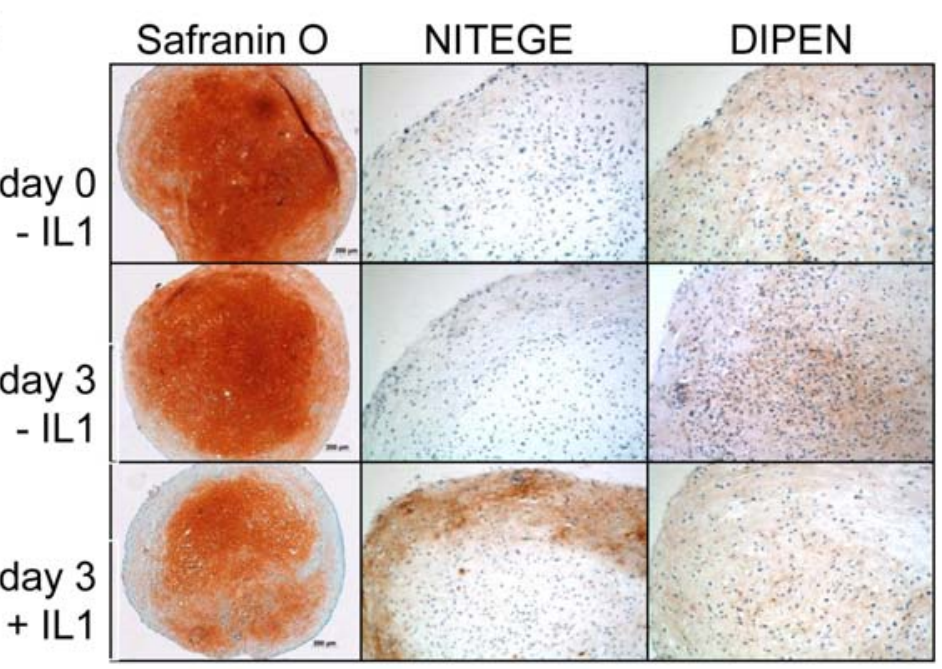

B

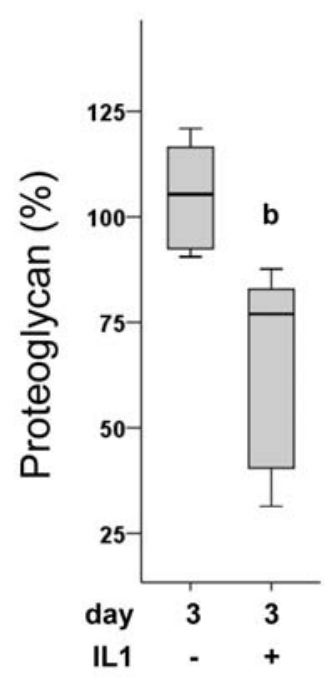

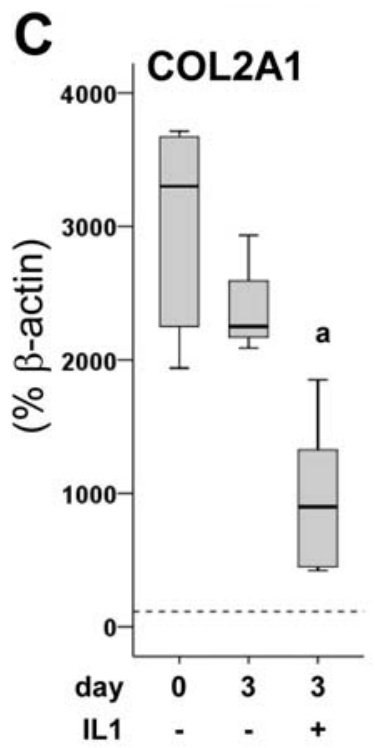
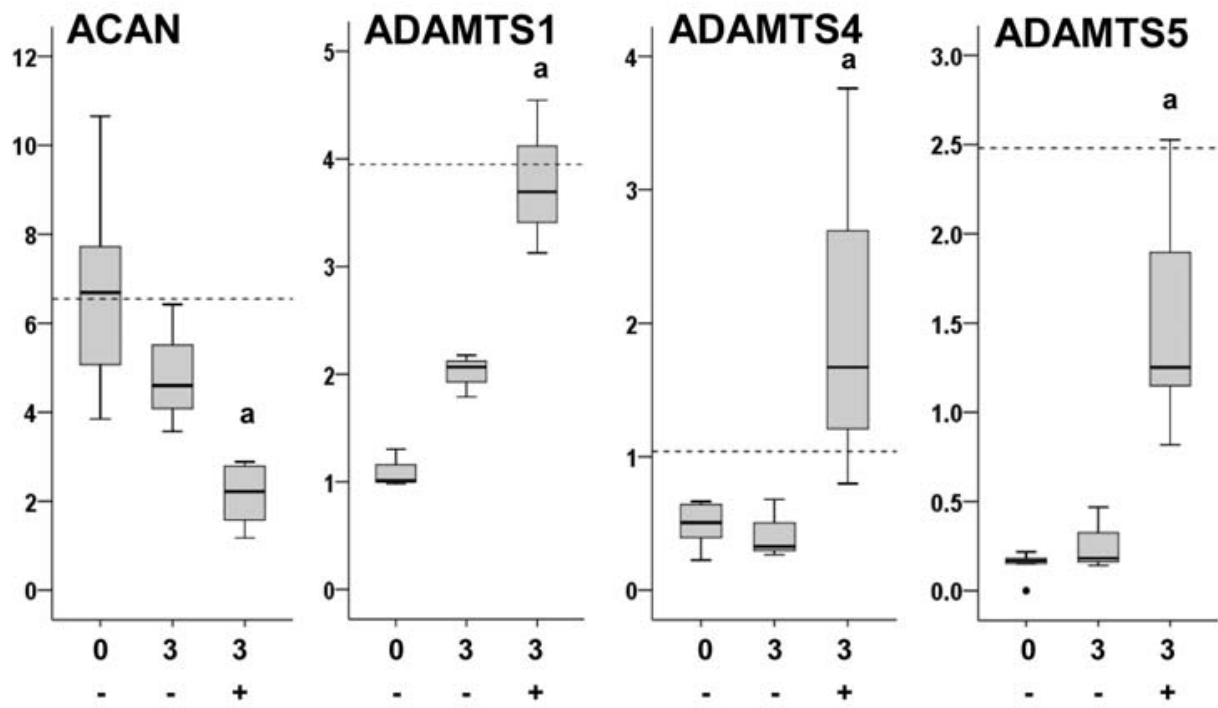

Fig. 4. Treatment of differentiated MSC pellets with IL1 $\beta$. Pellets after $42 \mathrm{~d}$ of chondrogenic differentiation were maintained $3 \mathrm{~d}$ in chondrogenic medium without TGF $\beta \pm$ supplementation with $10 \mathrm{ng} / \mathrm{mL} \operatorname{IL} 1 \beta(n=6)$. (A) Safranin O staining and immunohistochemical detection of NITEGE and DIPEN neo-epitopes in differentiated MSC pellets. Day 0 of treatment corresponds to day 42 of chondrogenesis. Differentiated pellets were maintained for $3 \mathrm{~d}$ without TGF $\beta$ (day 3; -IL1) or for $3 \mathrm{~d}$ without TGF $\beta$ and with IL1 $\beta$ (day 3; +IL1). Pellets from one representative donor are shown. (B) Proteoglycan content in 2 pellets per donor was quantified before and after IL1ß addition for $3 \mathrm{~d}$ from day 42 of chondrogenesis and values are expressed as percent of the mean amount of proteoglycan per donor before treatment $(n=6)$. $\mathbf{b}=$ significantly different from the day 0 and day 3 control groups $(p<0.05)$. (C) Gene expression levels as assessed by real time PCR are presented as relative values compared to the housekeeping gene $\beta$-actin in the respective samples. For each group, the median expression ratio in MSC samples is represented by a solid black line, the boxes represent the interquartile range (IQR) extending between the 25th and 75th percentile and the whiskers extend to a maximum of 1.5 IQR. Black dots are outlier values. Broken horizontal lines represent the median relative gene expression of the respective genes in normal cartilage samples $(n=6)$. $\mathbf{a}=$ significantly different from day $0(p<0.05)$.

and significant loss of proteoglycan to $66 \%$ of levels in control pellets (Figs. 4A and B). While MMP-cleavage of ACAN was not changed after IL1 $\beta$ treatment, as seen by the unchanged DIPEN neo-epitope, ADAMTS cleavage of aggrecan was clearly induced by IL1 $\beta$ (Fig. 4A).

In parallel, IL $1 \beta$ led to a more than 3-fold decrease of gene expression levels of COL2A1 and ACAN (Fig. 4C). Expression levels of ADAMTS1, 4 and 5 were significantly up-regulated (4.9-fold; 5.2-fold and 9.4-fold respectively), while no significant differences were found for ADAMTS9,
16 and furin (data not shown). Gene expression of ADAMTS 1 and ADAMTS5 after IL1 $\beta$ treatment reached levels close to cartilage (3.22\% and $1.48 \%$, respectively).

\section{Discussion}

We have shown that the ADAMTS with known aggrecanase activity can be classified in 2 groups showing different temporal expression patterns during chondrogenesis of 
MSCs. ADAMTS1 and 5 were down-regulated during differentiation while ADAMTS4, 9 and 16 were upregulated. Interestingly, the 2 classical and most potent aggrecanases, ADAMTS4 and ADAMTS5, were classified in 2 different groups. ADAMTS4, 9 and 16 showed low expression levels during expansion of MSCs and were up-regulated during chondrogenic differentiation, reaching levels equivalent to those in normal and OA cartilage for ADAMTS4 and 9, only to those in OA cartilage for ADAMTS16. The time point of the main shift of expression levels for ADAMTS9 and 16 was comparable to COL2A1 and ACAN, between day 7 and day 21 after induction of chondrogenesis. However, these two genes, albeit following a gene expression profile similar to that of classical chondrogenic differentiation markers, showed low expression levels and no induction by IL $1 \beta$ in differentiated MSCs so that further analysis would be necessary to determine whether they play any role in chondrogenesis.

Among the genes with up-regulated expression, the expression profile of ADAMTS4 was unusual as it showed an early peak of very high expression levels after induction of chondrogenesis. Studies have suggested that TGF $\beta$ has a short term stimulatory effect on ADAMTS4 expression, which is not observed for other ADAMTS. This has been shown in synovial tissue and fibroblast-like synoviocytes (Yamanishi et al., 2002) as well as in prostatic cancer cells (Cross et al., 2005). It is therefore possible that the expression peak at days 4 and 7 was due to the switch from a medium without TGF $\beta$ during expansion to a medium with TGF $\beta$ at day 0 . However, other signalling pathways are activated early after induction of chondrogenesis and could also be involved in the specific regulation pattern of ADAMTS4. In a study where chondrogenesis was induced with a BMP2-containing conditioned medium, ADAMTS4 was one among few genes showing an upregulation of expression after 2 days (Djouad et al., 2007). The ADAMTS4 up-regulation could thus be more generally linked to early chondrogenesis. We have shown previously that biglycan, COMP and decorin (Pelttari et al., 2006) which can be cleaved by ADAMTS4 (Melching et al., 2006; Kashigawa et al., 2004) are up-regulated as early as $2 \mathrm{~d}$ after induction of chondrogenesis in MSCs. Interestingly, all of these small proteoglycans modulate TGF $\beta$ signalling (Droguett et al., 2006; Ferdous et al., 2007; Haudenschild et al., 2011). ADAMTS4 could, thus, be co-regulated with these molecules in an early phase of chondrogenesis as a part of a feedback loop modulating availability of growth factors to the cells.

On the other hand, ADAMTS1 and ADAMTS5 showed higher expression levels during expansion and adaptation of MSCs to pellet culture than during chondroblast formation and differentiation (Dexheimer et al., 2012). Their down-regulation occurred during the main increase of ACAN expression between days 7 and 14. ADAMTS1 and 5 thus showed the highest expression levels during a phase when MSCs did not synthesise ACAN. This is in line with the detection of ADAMTS1 expression in the resting and proliferative zone of the growth plate of rats (Mitani et al., 2006). The function of these aggrecanases during early phases of differentiation remains unclear and is intriguing, especially for ADAMTS1 which showed high expression levels, comparable to those in normal cartilage. The aggrecanolytic ADAMTS also have other proteoglycan substrates, such as versican and members of the small leucine repeat proteoglycan family, cleavage of which are known to play a role in development and tissue morphogenesis (reviewed in Stanton et al., 2011). Whether the differential regulation of ADAMTS during MSC chondrogenesis is related to cleavage of proteoglycans other than aggrecan requires further investigation.

The chondrogenic induction of MSCs in vitro does not lead to the formation of articular-like chondrocytes but rather engages MSCs towards a hypertrophic differentiation stage characterised by the expression of markers such as collagen type X and MMP13 (Boeuf and Richter, 2010). This raises the question on how far the hypertrophic character of this differentiation process could be involved in the regulation of ADAMTS expression. Runx 2 is one of the main transcription factors regulating the expression of hypertrophy-associated genes (Solomon et al., 2008) and it strongly increased ADAMTS5 mRNA in human chondrosarcoma cells (Thirunavukkarasu et al., 2007). Studies in mice and rats suggested increased expression or activity of ADAMTS1, 4 and 5 in the hypertrophic zone of growth plate cartilage (Little et al., 2005; Mitani et al., 2006; Groma et al., 2011), while ADAMTS9 was expressed in the proliferating but not in the hypertrophic zone chondrocytes (Jungers et al., 2005). This suggests that a hypertrophic phenotype is characterised by high expression of ADAMTS1, 4 and 5 and low ADAMTS9. The decrease with time of ADAMTS1 and 5 expression and the increase of ADAMTS9 expression during in vitro chondrogenesis of MSCs are thus not consistent with a full endochondral differentiation of MSCs, albeit data from human growth plate are not available.

The phenotype of OA chondrocytes has been compared with a hypertrophic phenotype (Dreier, 2010), although the view of a generalised hypertrophy of OA chondrocytes is controversial (Brew et al., 2010). Of the genes up-regulated during chondrogenesis, ADAMTS9 and ADAMTS16 reached expression levels closer to OA cartilage at day 4, while expression was closer to normal cartilage for furin. Globally, the expression profile of the MSC pellets at day 42 cannot be classified as being closer to the normal or OA phenotypes of chondrocytes. Actually, differences of ADAMTS mRNA expression between OA and normal cartilage did not reach significance in our study, although it was almost the case for ADAMTS16 (5-fold higher in OA, $p=0.051$ ), in accordance with previous data (Kevorkian et al., 2004). Our study does, however, not support significantly lower ADAMTS1 levels in OA (Kevorkian et al., 2004), nor significantly higher expression of both ADAMTS4 and 5 in late but not early OA (Bau et al., 2002). Overall, data point out that ADAMTS expression may vary during the progression of the disease and that this may partly explain the divergent expression levels found in cartilage.

Although ADAMTS4, 9 and 16 were up-regulated during chondrogenesis, ADAMTS-induced cleavage of ACAN was not detectable during differentiation. Possibly 
ADAMTS activity is not required for proper differentiation but inactive proteins are produced and stored in the extracellular cartilage matrix for quick access in case of challenge. Indeed, exposure of pellets to IL $1 ß$ was able to rapidly induce aggrecanase enzyme activity, confirming that all required constituents to induce proteoglycan depletion are available. In contrast, MMP-induced cleavage of ACAN was detected without any further challenge and was found to occur as soon as MSC pellets initiate deposition of ACAN in the matrix. This is similar to what has been observed in normal juvenile cartilage, where MMP-cleaved, but not ADAMTS-cleaved, ACAN was found (Little et al., 1999). This suggests that ADAMTSinduced cleavage of ACAN may not be implicated in chondrogenic differentiation and that therefore the chondroprotective effect of aggrecanase inhibitors may not be associated with negative effects on chondrogenic and regenerative processes. It has indeed been shown that the inhibition of ADAMTS-induced cleavage of ACAN improves restoration of cartilage ACAN after its depletion in inflammatory joint disease (Little et al., 2007). In contrast, the inhibition of MMP-induced cleavage of ACAN did not allow cartilage regeneration and resulted in significantly worse cartilage damage in a surgical OA model (Little et al., 2007). This suggests that MMPinduced cleavage of ACAN may be protective in OA, which could be related to the essential function of MMPs during chondrogenesis (Bertram et al., 2009). Importantly, aggrecanase inhibition could have the advantage to allow a chondroprotective intervention which does not interfere with cartilage regeneration.

Exposure of MSC pellets to inflammatory conditions stimulated ADAMTS-induced cleavage of ACAN while MMP-cleaved ACAN was not affected. In terms of cleavage of ACAN, MSC pellets thus behave in a similar way to normal young articular cartilage (Little et al., 1999). IL1 $\beta$ induced an up-regulation of ADAMTS1, 4 and 5 and a down-regulation of COL2A1 and ACAN expression. The down-regulation of extracellular matrix genes by IL1 $\beta$ treatment is in line with observations for articular chondrocytes (Fan et al., 2005) and the known inhibitory activity of IL1 $\beta$ on in vitro chondrogenesis of MSCs (Felka et al., 2009). On the other hand, our data show that activation of ADAMTS by IL $1 \beta$ treatment could be partially due to transcriptional regulation of the aggrecanases, especially as these include the 2 classical human aggrecanases thought to be the major contributors to ACAN depletion in humans (Song et al., 2007). Interestingly, ADAMTS4 and 5 showed very different patterns of gene expression during chondrogenesis of MSCs, but IL1 $\beta$ treatment resulted in a marked induction of the expression of both genes. This is different from what has been reported for human articular cartilage or articular chondrocytes, where in most studies IL1 $\beta$ was found to induce expression of ADAMTS4 but not ADAMTS5 (Fosang and Rogerson, 2010). Thus, while ADAMTS4 appears to be expressed in differentiated MSCs at similar levels as in cartilage and to be inducible under identical conditions, ADAMTS5 expression and inducibility in MSCs differed from articular cartilage.

\section{Conclusions}

In summary, we have shown that although MSCs induce the expression of the genes for ADAMTS4, 9 and 16 during chondrogenesis, no ADAMTS-induced cleavage of ACAN but rather an MMP mediated neoepitope formation occurs. ADAMTS-induced cleavage can however be stimulated by an inflammatory signal in chondrogenic MSCs like in articular cartilage. ADAMTS-induced cleavage of ACAN, thus, appears not to be critical for chondrogenic differentiation of MSCs, while it plays an essential role for ACAN depletion upon inflammatory signals. These results suggest that aggrecanase inhibition could be beneficial in degenerative diseases, not only based on its protective effects on the matrix but also because it does not interfere with chondrogenesis, and thus may allow regenerative processes based on endogenous progenitor cells to take place.

\section{Acknowledgements}

This work was financially supported by The German Research Foundation DFG Ri707-/7-1, the Orthopaedic University Hospital Heidelberg, the University of Sydney, the NSW Office of Science and Medical Research through the Kolling Institute of Medical Research and the National Health and Medical Research Council of Australia. The authors would like to thank Anika Krase for her precious help, Rosalie Bock, Michaela Burkhardt, Birgit Frey and Susan Smith for excellent technical assistance and Simone Gantz for statistical support.

\section{References}

Aigner T, McKenna L (2002) Molecular pathology and pathobiology of osteoarthritic cartilage. Cell Mol Life Sci 59: 5-18.

Bau B, Gebhard PM, Haag J, Knorr T, Bartnik E, Aigner $\mathrm{T}$ (2002) Relative messenger RNA expression profiling of collagenases and aggrecanases in human articular chondrocytes in vivo and in vitro. Arthritis Rheum 46: 2648-2657.

Bertram H, Boeuf S, Wachters J, Boehmer S, Heisel C, Hofmann MW, Piecha D, Richter W (2009) Matrix metalloprotease inhibitors suppress initiation and progression of chondrogenic differentiation of mesenchymal stromal cells in vitro. Stem Cells Dev 18: 881-892.

Björnsson S (1993) Simultaneous preparation and quantitation of proteoglycans by precipitation with alcian blue. Anal Biochem 210: 282-291.

Boeuf S, Richter W (2010) Chondrogenesis of mesenchymal stem cells: role of tissue source and inducing factors. Stem Cell Res Ther 1: 31.

Brew CJ, Clegg PD, Boot-Handford RP, Andrew JG, Hardingham T (2010) Gene expression in human chondrocytes in late osteoarthritis is changed in both fibrillated and intact cartilage without evidence of generalised chondrocyte hypertrophy. Ann Rheum Dis 69: 234-240. 
Cross NA, Chandrasekharan S, Jokonya N, Fowles A, Hamdy FC, Buttle DJ, Eaton CL (2005) The expression and regulation of ADAMTS-1, $-4,-5,-9$ and -15 , and TIMP-3 by TGFbeta1 in prostate cells: relevance to the accumulation of versican. Prostate 63: 269-275.

Dexheimer V, Frank S, Richter W (2012) Proliferation as a requirement for in vitro chondrogenesis of human mesenchymal stem cells. Stem Cells Dev doi:10.1089/ scd2011.0670.

Djouad F, Delorme B, Maurice M, Bony C, Apparailly F, Louis-Plence P, Canovas F, Charbord P, Noël D, Jorgensen C (2007) Microenvironmental changes during differentiation of mesenchymal stem cells towards chondrocytes. Arthritis Res Ther 9: R33.

Dreier R (2010) Hypertrophic differentiation of chondrocytes in osteoarthritis: the developmental aspect of degenerative joint disorders. Arthritis Res Ther 12: 216.

Droguett R, Cabello-Verrugio C, Riquelme C, Brandan E (2006) Extracellular proteoglycans modify TGF-beta bio-availability attenuating its signaling during skeletal muscle differentiation. Matrix Biol 25: 332-341.

Fan Z, Bau B, Yang H, Soeder S, Aigner T (2005) Freshly isolated osteoarthritic chondrocytes are catabolically more active than normal chondrocytes, but less responsive to catabolic stimulation with interleukin-1beta. Arthritis Rheum 52: 136-143.

Felka T, Schäfer R, Schewe B, Benz K, Aicher WK (2009) Hypoxia reduces the inhibitory effect of IL-1beta on chondrogenic differentiation of FCS-free expanded MSC. Osteoarthritis Cartilage 17: 1368-1376.

Ferdous Z, Wei VM, Iozzo R, Höök M, GrandeAllen KJ (2007) Decorin-transforming growth factorinteraction regulates matrix organization and mechanical characteristics of three-dimensional collagen matrices. J Biol Chem 282: 35887-35898.

Fosang AJ, Little CB (2008) Drug insight: aggrecanases as therapeutic targets for osteoarthritis. Nat Clin Pract Rheumatol 4: 420-427.

Fosang AJ, Rogerson FM (2010) Identifying the human aggrecanase. Osteoarthritis Cartilage 18: 1109-1116.

Groma G, Grskovic I, Schael S, Ehlen HW, Wagener R, Fosang A, Aszodi A, Paulsson M, Brachvogel B, Zaucke F (2011) Matrilin-4 is processed by ADAMTS-5 in late Golgi vesicles present in growth plate chondrocytes of defined differentiation state. Matrix Biol 30: 275-280.

Haudenschild DR, Hong E, Yik JH, Chromy B, Mörgelin M, Snow KD, Acharya C, Takada Y, Di Cesare PE (2011) Enhanced activity of transforming growth factor $\beta 1$ (TGF- $\beta 1$ ) bound to cartilage oligomeric matrix protein. J Biol Chem 286: 43250-43258.

Haynesworth SE, Goshima J, Goldberg VM, Caplan AI (1992) Characterization of cells with osteogenic potential from human marrow. Bone 13: 81-88.

Hennig T, Lorenz H, Thiel A, Goetzke K, Dickhut A, Geiger F, Richter W (2007) Reduced chondrogenic potential of adipose tissue derived stromal cells correlates with an altered TGFbeta receptor and BMP profile and is overcome by BMP-6. J Cell Physiol 211: 682-691.

Jones GC, Riley GP (2005) ADAMTS proteinases: a multi-domain, multi-functional family with roles in extracellular matrix turnover and arthritis. Arthritis Res Ther 7: 160-169.

Jungers KA, Le Goff C, Somerville RP, Apte SS (2005) Adamts9 is widely expressed during mouse embryo development. Gene Expr Patterns 5: 609-617.

Kashiwagi M, Enghild JJ, Gendron C, Hughes C, Caterson B, Itoh Y, Nagase H (2004) Altered proteolytic activities of ADAMTS-4 expressed by C-terminal processing. J Biol Chem 279: 10109-10119.

Kevorkian L, Young DA, Darrah C, Donell ST, Shepstone L, Porter S, Brockbank SM, Edwards DR, Parker AE, Clark IM (2004) Expression profiling of metalloproteinases and their inhibitors in cartilage. Arthritis Rheum 50: 131-141.

Koo BH, Longpre JM, Somerville RP, Alexander JP, Leduc R, Apte SS (2006) Cell-surface processing of proADAMTS9 by furin. J Biol Chem 281: 12485-12494.

Little CB, Fosang AJ (2010) Is cartilage matrix breakdown an appropriate therapeutic target in osteoarthritis--insights from studies of aggrecan and collagen proteolysis? Curr Drug Targets 11: 561-575.

Little CB, Flannery CR, Hughes CE, Mort JS, Roughley PJ, Dent C, Caterson B (1999) Aggrecanase versus matrix metalloproteinases in the catabolism of the interglobular domain of aggrecan in vitro. Biochem J 344: 61-68.

Little CB, Mittaz L, Belluoccio D, Rogerson FM, Campbell IK, Meeker CT, Bateman JF, Pritchard MA, Fosang AJ (2005) ADAMTS-1-knockout mice do not exhibit abnormalities in aggrecan turnover in vitro or in vivo. Arthritis Rheum 52: 1461-1472.

Little CB, Meeker CT, Golub SB, Lawlor KE, Farmer PJ, Smith SM, Fosang AJ (2007) Blocking aggrecanase cleavage in the aggrecan interglobular domain abrogates cartilage erosion and promotes cartilage repair. J Clin Invest 117: 1627-1636.

Longpre JM, Leduc R (2004) Identification of prodomain determinants involved in ADAMTS-1 biosynthesis. J Biol Chem 279: 33237-33245.

Longpre JM, McCulloch DR, Koo BH, Alexander JP, Apte SS, Leduc R (2009) Characterization of proADAMTS5 processing by proprotein convertases. Int J Biochem Cell Biol 41: 1116-1126.

Malfait AM, Liu RQ, Ijiri K, Komiya S, Tortorella MD (2002) Inhibition of ADAM-TS4 and ADAM-TS5 prevents aggrecan degradation in osteoarthritic cartilage. J Biol Chem 277: 22201-22208.

Melching LI, Fisher WD, Lee ER, Mort JS, Roughley PJ (2006) The cleavage of biglycan by aggrecanases. Osteoarthritis Cartilage 14: 1147-1154.

Mitani H, Takahashi I, Onodera K, Bae JW, Sato T, Takahashi N, Sasano Y, Igarashi K, Mitani H (2006) Comparison of age-dependent expression of aggrecan and ADAMTSs in mandibular condylar cartilage, tibial growth plate, and articular cartilage in rats. Histochem Cell Biol 126: 371-380.

Pelttari K, Winter A, Steck E, Goetzke K, Hennig T, Ochs BG, Aigner T, Richter W (2006) Premature induction of hypertrophy during in vitro chondrogenesis of human mesenchymal stem cells correlates with calcification and vascular invasion after ectopic transplantation in SCID mice. Arthritis Rheum 54: 3254-3266. 
Porter S, Clark IM, Kevorkian L, Edwards DR (2005) The ADAMTS metalloproteinases. Biochem J 386: 15-27.

Reyes M, Lund T, Lenvik T, Aguiar D, Koodie L, Verfaillie CM (2001) Purification and ex vivo expansion of postnatal human marrow mesodermal progenitor cells. Blood 98: 2615-2625.

Smith MM, Sakurai G, Smith SM, Young AA, Melrose J, Stewart CM, Appleyard RC, Peterson JL, Gillies RM, Dart AJ, Sonnabend DH, Little CB (2008) Modulation of aggrecan and ADAMTS expression in ovine tendinopathy induced by altered strain. Arthritis Rheum 58: 1055-1066.

Solomon LA, Bérubé NG, Beier F (2008) Transcriptional regulators of chondrocyte hypertrophy. Birth Defects Res C Embryo Today 84: 123-130.

Song RH, Tortorella MD, Malfait AM, Alston JT, Yang Z, Arner EC, Griggs DW (2007) Aggrecan degradation in human articular cartilage explants is mediated by both ADAMTS-4 and ADAMTS-5. Arthritis Rheum 56: 575585.

Stanton H, Rogerson FM, East CJ, Golub SB, Lawlor KE, Meeker CT, Little CB, Last K, Farmer PJ, Campbell IK, Fourie AM, Fosang AJ (2005) ADAMTS5 is the major aggrecanase in mouse cartilage in vivo and in vitro. Nature 434: 648-652.

Stanton H, Melrose J, Little CB, Fosang AJ (2011) Proteoglycan degradation by the ADAMTS family of proteinases. Biochim Biophys Acta 1812: 1616-1629.

Sumer EU, Qvist P, Tanko LB (2007) Matrix metalloproteinase and aggrecanase generated aggrecan fragments: implications for the diagnostics and therapeutics of destructive joint diseases. Drug Dev Res 68: 1-13.

Thirunavukkarasu K, Pei Y, Wei T (2007) Characterization of the human ADAMTS-5 (aggrecanase-2) gene promoter. Mol Biol Rep 34: 225-231.

Wang P, Tortorella M, England K, Malfait AM, Thomas G, Arner EC, Pei D (2004) Proprotein convertase furin interacts with and cleaves pro-ADAMTS4 (Aggrecanase-1) in the trans-Golgi network. J Biol Chem 279: 15434-15440.

Winter A, Breit S, Parsch D, Benz K, Steck E, Hauner H, Weber RM, Ewerbeck V, Richter W (2003) Cartilagelike gene expression in differentiated human stem cell spheroids: a comparison of bone marrow-derived and adipose tissue-derived stromal cells. Arthritis Rheum 48: 418-429.

Yamanishi Y, Boyle DL, Clark M, Maki RA, Tortorella MD, Arner EC, Firestein GS (2002) Expression and regulation of aggrecanase in arthritis: the role of TGF-beta. J Immunol 168: 1405-1412.

Zeng W, Corcoran C, Collins-Racie LA, LaVallie ER, Morris EA, Flannery CR (2006) Glycosaminoglycanbinding properties and aggrecanase activities of truncated ADAMTSs: comparative analyses with ADAMTS-5, -9, -16 and -18. Biochim Biophys Acta 1760: 517-254.

\section{Discussion with Reviewers}

Reviewer II: Given the discrepancy in this manuscript and also by now shown by many others between mRNA and protein/activity, how valuable are PCRs as stand-alone read-out parameters in in vitro research?
Authors: PCR as a stand-alone read-out parameter is only valuable in cases where a clear correlation of gene expression to protein synthesis or activity has been demonstrated previously. Many regulation mechanisms can interfere and regulate protein expression at a posttranscriptional level, including mRNAi which has been discovered as playing important roles in most cellular processes. Protein activity is furthermore regulated by activation mechanisms. PCR analysis and its correlation to protein expression and activity data is, therefore, important to evaluate the impact of such post-transcriptional regulatory mechanisms. Without further analysis, gene expression levels alone do not allow conclusions on protein expression and even less on protein activity. Our study, consequently, includes histological assessment of ADAMTS enzyme activity by detection of aggrecan neoepitopes generated during cleavage, and highlights once more that PCR cannot be used as a stand-alone read-out parameter in the study of proteases.

Reviewer II: Historically, it has been difficult to selectively inhibit specific MMPs, and your previous studies have shown MMPs are needed for chondrogenesis. What is known about the ability to inhibit specific ADAMTS and do these inhibitors have a cross-reactivity with MMPs?

Authors: Given the central role of the major aggrecanases (ADAMTS-4 and 5) in cartilage aggrecan degradation and $\mathrm{OA}$, there has been extensive investigation into the development of selective inhibitors particularly by the pharmaceutical industry. A number of compounds have been reported with various levels of specificity for the aggrecanases over other metalloproteinases (MMPs and ADAMs), and for ADAMTS-4 versus ADAMTS-5 (Shiozaki et al., 2011; Shieh et al., 2011; Lim et al., 2010; Tortorella et al., 2009; Shiozaki et al., 2009; Gilbert et al., 2008; Bursavich et al., 2007). To date, the effect of these selective aggrecanase inhibitors on MSC differentiation have not, to the best of our knowledge, been examined. Given the temporal regulation of various aggrecanolytic ADAMTS that we have observed during in vitro chondrogenesis, such studies would be very interesting.

Reviewer II: Is there any evidence that ADAMTSs are mechanosensitive and that could trigger their misregulation during OA? In your discussion you mention that ADAMTSs may be "stored in the ECM": is there any data if or where they may localise?

Authors: There is evidence that expression and/or activity, of ADAMTS-4 and 5 can be regulated by mechanical loading in chondrocytes (Tetsunaga et al., 2011; Ding et al., 2010; Wheeler et al., 2009). Whether this is a direct effect through mechanosensitive elements in the upstream of the ADAMTS genes, or is secondary to mechanotransduction of other regulatory proteins such as IL-1, runx2, NF-kB or CITED2 (Leong et al., 2011) remains to be elucidated. Nevertheless, changes in mechanical loading of cartilage may play a primary role in regulating cartilage breakdown in OA.

The ADAMTS bind to the ECM largely through interaction of their C-terminal domains with various matrix proteins and glycosaminoglycans (Stanton et al., 
2011; Nagase et al., 2008; Gao et al., 2004; Kuno and Matsushima, 1998; Kuno et al., 1999). There is evidence for accumulation of pro-ADAMTS in the pericellular region and further in the cartilage matrix, which can be activated by pro-protein convertases (Pratta et al., 2003; Malfait et al., 2008). How this matrix binding and activation is regulated during chondrogenesis by MSCs requires further investigation.

\section{Additional References}

Bursavich MG, Gilbert AM, Lombardi S, Georgiadis KE, Reifenberg E, Flannery CR, Morris EA (2007) 5'-Phenyl-3'H-spiro[indoline-3,2'-[1,3,4]thiadiazol]-2one inhibitors of ADAMTS-5 (aggrecanase-2). Bioorg Med Chem Lett 17: 5630-5633.

Ding L, Heying E, Nicholson N, Stroud NJ, Homandberg GA, Buckwalter JA, Guo D, Martin JA (2010) Mechanical impact induces cartilage degradation via mitogen activated protein kinases. Osteoarthritis Cartilage 18: 1509-1517.

Gao G, Plaas A, Thompson VP, Jin S, Zuo F, Sandy JD (2004) ADAMTS4 (aggrecanase-1) activation on the cell surface involves C-terminal cleavage by glycosylphosphatidyl inositol-anchored membrane type 4-matrix metalloproteinase and binding of the activated proteinase to chondroitin sulfate and heparan sulfate on syndecan-1. J Biol Chem 279: 10042-10051.

Gilbert AM, Bursavich MG, Lombardi S, Georgiadis KE, Reifenberg E, Flannery CR, Morris EA (2008) N-((8hydroxy-5-substituted-quinolin-7-yl)(phenyl)methyl)-2phenyloxy/amino-acetamide inhibitors of ADAMTS-5 (Aggrecanase-2). Bioorg Med Chem Lett 18: 6454-6457.

Kuno K, Matsushima K (1998) ADAMTS-1 protein anchors at the extracellular matrix through the thrombospondin type I motifs and its spacing region. J Biol Chem 273: 13912-13917.

Kuno K, Terashima Y, Matsushima K (1999) ADAMTS-1 is an active metalloproteinase associated with the extracellular matrix. J Biol Chem 274: 18821-18826.

Leong DJ, Hardin JA, Cobelli NJ, Sun HB (2011) Mechanotransduction and cartilage integrity. Ann N Y Acad Sci 1240: 32-37.

Lim NH, Kashiwagi M, Visse R, Jones J, Enghild JJ, Brew K, Nagase H (2010) Reactive-site mutants of N-TIMP-3 that selectively inhibit ADAMTS-4 and ADAMTS-5: biological and structural implications. Biochem J 431: 113-122.
Malfait AM, Arner EC, Song RH, Alston JT, Markosyan S, Staten N, Yang Z, Griggs DW, Tortorella MD (2008) Proprotein convertase activation of aggrecanases in cartilage in situ. Arch Biochem Biophys 478: 43-51.

Nagase H, Fushimi K (2008) Elucidating the function of non catalytic domains of collagenases and aggrecanases. Connect Tissue Res 49: 169-174.

Pratta MA, Scherle PA, Yang G, Liu RQ, Newton $\mathrm{RC}$ (2003) Induction of aggrecanase 1 (ADAM-TS4) by interleukin-1 occurs through activation of constitutively produced protein. Arthritis Rheum 48: 119-133.

Shieh HS, Tomasselli AG, Mathis KJ, Schnute ME, Woodard SS, Caspers N, Williams JM, Kiefer JR, Munie G, Wittwer A, Malfait AM, Tortorella MD (2011) Structure analysis reveals the flexibility of the ADAMTS-5 active site. Protein Sci 20: 735-744.

Shiozaki M, Maeda K, Miura T, Ogoshi Y, Haas J, Fryer AM, Laird ER, Littmann NM, Andrews SW, Josey JA, Mimura T, Shinozaki Y, Yoshiuchi H, Inaba T (2009) Novel $\mathrm{N}$-substituted 2-phenyl-1-sulfonylamino-cyclopropane carboxylates as selective ADAMTS-5 (Aggrecanase-2) inhibitors. Bioorg Med Chem Lett 19: 1575-1580.

Shiozaki M, Maeda K, Miura T, Kotoku M, Yamasaki T, Matsuda I, Aoki K, Yasue K, Imai H, Ubukata M, Suma A, Yokota M, Hotta T, Tanaka M, Hase Y, Haas J, Fryer AM, Laird ER, Littmann NM, Andrews SW, Josey JA, Mimura T, Shinozaki Y, Yoshiuchi H, Inaba T (2011) Discovery of (1S,2R,3R)-2,3-dimethyl-2-phenyl-1-sulfamidocycloprop anecarboxylates: novel and highly selective aggrecanase inhibitors. J Med Chem 54: 2839-2863.

Tetsunaga T, Nishida K, Furumatsu T, Naruse K, Hirohata S, Yoshida A, Saito T, Ozaki T (2011) Regulation of mechanical stress-induced MMP-13 and ADAMTS-5 expression by RUNX-2 transcriptional factor in SW1353 chondrocyte-like cells. Osteoarthritis Cartilage 19: 222232.

Tortorella MD, Tomasselli AG, Mathis KJ, Schnute ME, Woodard SS, Munie G, Williams JM, Caspers N, Wittwer AJ, Malfait AM, Shieh HS (2009) Structural and inhibition analysis reveals the mechanism of selectivity of a series of aggrecanase inhibitors. J Biol Chem 284: 24185-24191.

Wheeler CA, Jafarzadeh SR, Rocke DM, Grodzinsky AJ (2009) IGF-1 does not moderate the time-dependent transcriptional patterns of key homeostatic genes induced by sustained compression of bovine cartilage. Osteoarthritis Cartilage 17: 944-952. 